\title{
Longitudinal phase-space manipulation of ellipsoidal electron bunches in realistic fields
}

\author{
S. B. van der Geer, ${ }^{*}$ M. J. de Loos, ${ }^{\dagger}$ T. van Oudheusden, W. P. E. M. op 't Root, M. J. van der Wiel, and O. J. Luiten \\ Eindhoven University of Technology, Center for Plasma Physics and Beam Technology, \\ P.O. Box 513, 5600 MB Eindhoven, The Netherlands
}

(Received 25 January 2006; published 26 April 2006)

\begin{abstract}
Since the recent publication of a practical recipe to create "pancake" electron bunches which evolve into uniformly filled ellipsoids, a number of papers have addressed both an alternative method to create such ellipsoids as well as their behavior in realistic fields. So far, the focus has been on the possibilities to preserve the initial "thermal" transverse emittance. This paper addresses the linear longitudinal phase space of ellipsoidal bunches. It is shown that ellipsoidal bunches allow ballistic compression at subrelativistic energies, without the detrimental effects of nonlinear space-charge forces. This in turn eliminates the need for the large correlated energy spread normally required for longitudinal compression of relativistic particle beams, while simultaneously avoiding all problems related to magnetic compression. Furthermore, the linear space-charge forces of ellipsoidal bunches can be used to reduce the remaining energy spread even further, by carefully choosing the beam transverse size, in a process that is essentially the time-reversed process of the creation of an ellipsoid at the cathode. The feasibility of compression of ellipsoidal bunches is illustrated with a relatively simple setup, consisting of a half-cell S-band photogun and a two-cell booster compressor. Detailed GPT simulations in realistic fields predict that $100 \mathrm{pC}$ ellipsoidal bunches can be ballistically compressed to $100 \mathrm{fs}$, at a transverse emittance of $0.7 \mu \mathrm{m}$, with a final energy of $3.7 \mathrm{MeV}$ and an energy spread of only $50 \mathrm{keV}$.
\end{abstract}

DOI: 10.1103/PhysRevSTAB.9.044203

PACS numbers: 29.27.Ac, 29.27.Bd, 41.75.Ht, 41.85.Lc

\section{INTRODUCTION}

The most important process limiting the quality of pulsed, high-brightness electron beams is the unrecoverable distortion of the six-dimensional (6D) phase-space distribution due to nonlinear space-charge forces. The only charged particle distributions which are characterized by linear space-charge fields in all dimensions, and therefore theoretically do not suffer from deteriorating effects of space-charge forces, are uniformly filled ellipsoidal electron bunches, also known as "waterbag" bunches [1,2]. For a long time they have been known to be the ideal objects for controlled, high-brightness charged particle acceleration, but they were never considered a practical possibility. In 1997 Serafini was the first to propose that waterbag bunches may be realized in practice by using high-gradient radio-frequency (rf) photoguns, operated in the space-charge driven "blowout" regime [3]. The square femtosecond temporal laser profile, required according to Ref. [3], is however not practically feasible, and the proposed parabolic radial profile does not lead to a true waterbag bunch. In 2004 Luiten et al. showed that waterbag bunches can be created in practice because any temporal laser profile is acceptable as long as the laser pulse is sufficiently short, and that the ideal initial radial profile is a half circle [4]. Detailed general particle tracer (GPT) [5]

\footnotetext{
*Electronic address: s.b.van.der.geer@tue.nl
}

${ }^{\dagger}$ Also at Pulsar Physics, The Netherlands. simulations convincingly demonstrated the possibility of producing true waterbag bunches in a uniform acceleration field. In Ref. [6] it was shown that waterbag bunches can also be created in the nonuniform fields of an rf photogun, including magnetic focusing, and that the initial transverse emittance can be fully recovered. Subsequently several papers appeared further discussing the advantages of waterbag bunches [7] and the combination with emittance compensation [8].

Because of the almost perfectly linear longitudinal phase-space characteristics, waterbag bunches are the ideal starting point for all kinds of bunch compression schemes. In this paper we focus on the longitudinal phase-space behavior of waterbag bunches in realistic fields, in particular, the realization of high currents combined with lowenergy spread. Contrary to all other charge density distributions, waterbag bunches do not require fast acceleration to relativistic speeds in order to prevent degradation of bunch quality due to space-charge forces. This allows longitudinal ballistic compression at sub or mildly relativistic energies, which is much more efficient because far less energy spread is required compared to the high-energy alternatives used in present day systems [9]. In addition, compressing ellipsoidal bunches allows a further reduction of the energy spread by making use of the fact that the sign of the slope of the distribution in longitudinal phase space due to space-charge forces is opposite to the sign of the slope required for compression: As the bunch is compressed, kinetic energy is converted back again into potential energy. 
The advantages of ballistic compression of ellipsoidal bunches at relatively low energy are illustrated in this paper by detailed GPT simulation results of a relatively simple setup. In the proposed setup, waterbag bunches are created at $1 \mathrm{MeV}$ in a half-cell S-band rf cavity. After expansion, the bunches are sent through a 2-cell S-band rf cavity, acting both as buncher and booster, which increases the energy to $4 \mathrm{MeV}$ and produces a longitudinal focus $1.5 \mathrm{~m}$ downstream the cathode plane. The setup, comparable in size and complexity to a single rf photogun [10], produces bunches with parameters that, apart from final energy, can compete with large accelerator facilities: in the order of 100 fs duration, $1 \mathrm{kA}$ peak current and a transverse emittance of about $1 \mu \mathrm{m}$.

It is clear that ballistic compression of ellipsoidal bunches allows a broad range of tabletop devices to be constructed that depend on ultrashort high-brightness electron bunches where the final energy is not crucial. One promising example is a compact high-power broadband terahertz source based on coherent transition radiation (CTR). CTR is emitted when an ultrashort relativistic bunch passes through a conducting foil, but the energy is not crucial because the total photon yield only scales with the logarithm of the kinetic energy [11]. Another research area that could greatly benefit from the availability of mildly relativistic ultrashort high-brightness bunches is laser-wakefield acceleration with external injection $[12,13]$ : As long as the energy of the electron bunch is sufficiently high to be trapped by the plasma-wakefield, additional injection energy will only marginally increase the dephasing length.

Apart from these existing applications, new applications appear at the horizon which actually require mildly relativistic, or even nonrelativistic energies. Compressed ellipsoidal bunches of a few $\mathrm{MeV}$ can, for example, be used for "pump-probe" pulsed radiolysis experiments on $100 \mathrm{fs}$ time scales, to study rapid chemical reaction dynamics [14]. At even lower energy, the same compression process is ideal for time-resolved ultrafast electron diffraction experiments [15].

The relatively simple setup produces bunches with a brightness of $B=0.14 \mathrm{~A} \mathrm{\mu m}^{-2} \mathrm{sr}^{-1} \mathrm{keV}^{-1}$, i.e., an order of magnitude better than the best velocity bunching results presented in Ref. [9]. However, as will be shown in this paper, there are still many orders of magnitude to be gained, and the results presented are only a first step towards the distant goal of full recovery of the intrinsic brightness of the photoemission process.

The remainder of this paper is organized as follows: First Sec. II discusses brightness definitions and limits. Subsequently Sec. III presents an introduction to brightness degradation mechanisms, while Sec. IV briefly summarizes our recipe to create waterbag bunches in an $\mathrm{rf}$ photogun. Section V contains a description of the proposed setup and detailed simulation results. Finally, Sec. VI ends with a discussion and future outlook.

\section{PARTICLE BEAM BRIGHTNESS: DEFINITIONS AND LIMITS}

The fundamental characterization of a collisionless pulsed charged particle beam is given in terms of the local phase-space density distribution $f(\vec{r}, \vec{p})$, normalized to the number of particles $N=\int f(\vec{r}, \vec{p}) d^{3} r d^{3} p$. The local phase-space density is Lorentz invariant, and a constant along the particle trajectories during transport and acceleration according to Liouville's theorem. The quality of particle beams, however, is usually expressed in terms of the (6D) brightness $B$, which is the current density per unit solid angle and per unit energy spread. We therefore define the local $6 \mathrm{D}$ brightness in a Lorentz-invariant way by

$$
B(\vec{r}, \vec{p}) \equiv e m^{2} c^{2} f(\vec{r}, \vec{p}),
$$

with $e$ the elementary charge, $m$ the electron mass, and $c$ the speed of light. In practice only bunch or slice-averaged quantities are measured. Following Brau's treatment in Ref. [16], it can easily be shown that for a particle bunch with a $6 \mathrm{D}$ Gaussian phase-space distribution the (peak) 6D brightness $B \equiv B(0,0)$ at the center of the bunch is given by

$$
B=\frac{1}{m c} \frac{N e}{(2 \pi)^{3} \epsilon_{x} \epsilon_{y} \epsilon_{z}} .
$$

Here the root mean square (rms) normalized emittance $\epsilon_{x}$ is given by

$$
\epsilon_{x}=\frac{1}{m c} \sqrt{\left\langle x^{2}\right\rangle\left\langle p_{x}^{2}\right\rangle-\left\langle x p_{x}\right\rangle^{2}}
$$

with $p_{x}=\gamma m v_{x}$ the momentum in the $x$ direction, $\gamma=$ $\left(1-v^{2} / c^{2}\right)^{-1 / 2}$ the Lorentz factor, and $v$ the particle velocity. The brackets $\langle\cdots\rangle$ denote averaging over the entire bunch. The emittances $\epsilon_{y}$ and $\epsilon_{z}$ are defined analogously. In practice, the exact phase-space distribution is not known. If we assume, however, that the $x, y$, and $z$ directions are decoupled, then definition (2) supplies us with a practical estimate of peak $6 \mathrm{D}$ brightness.

For many applications the peak current and the transverse normalized emittances are the most important parameters, while the energy spread is less critical. For this reason the most often used figure of merit for overall bunch quality is the transverse brightness $B_{\perp}$, which is defined by [16]:

$$
B_{\perp}=\frac{I}{4 \pi^{2} \epsilon_{x} \epsilon_{y}},
$$

where $I$ is the peak current. If the distribution in $z-p_{z}$ space is Gaussian, then the transverse brightness $B_{\perp}$ is simply proportional to the product of the $6 \mathrm{D}$ brightness $B$ 
and the rms energy spread:

$$
B_{\perp}=B \sigma_{E} \sqrt{2 \pi},
$$

where $\sigma_{E}=m c^{2} \sigma_{\gamma}=m c^{2} \sqrt{\left\langle(\gamma-\langle\gamma\rangle)^{2}\right\rangle}$. Note that the transverse brightness is not a Lorentz-invariant quantity and is therefore not suited for making statements about the ultimate beam quality that can be reached.

To illustrate this, let us consider the case of photoemission from a copper surface of a $100 \mathrm{pC}$ electron bunch with a 30 fs UV laser pulse, in an acceleration field of $100 \mathrm{MV} / \mathrm{m}$. Assuming isotropic photoemission with an average kinetic energy $k T=0.5 \mathrm{eV}$, the initial rms momentum spread in any direction is given by $\sigma_{p_{x}} / m c=$ $\sigma_{p_{y}} / m c=\sqrt{2} \sigma_{p_{z}} / m c=\sqrt{k T / m c^{2}}=1 \times 10^{-3}$. For a beam radius of $1 \mathrm{~mm}$, i.e., $\sigma_{x} \approx 0.5 \mathrm{~mm}$, the initial $\mathrm{rms}$ transverse emittance thus amounts to $\epsilon_{x} \approx 0.5 \mu \mathrm{m}$. The rms bunch length immediately after photoemission is approximately given by $\sigma_{z} \approx 10 \mathrm{~nm}$, assuming sufficient flatness of the cathode surface. This gives rise to an upper bound for the initial rms longitudinal emittance of $\epsilon_{z}=$ $5 \times 10^{-6} \mu \mathrm{m}$. Using Eq. (2) we thus arrive at a $6 \mathrm{D}$ source

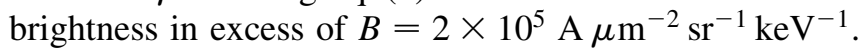
We would like to stress that this value can in principle be maintained during transport and acceleration, but in practice it is not reached by far in rf photoguns.

Making use of waterbag bunches [4,6,7], possibly combined with emittance compensation schemes $[8,17]$, rf photoguns are able to almost fully recover the initial transverse emittance. The longitudinal emittance, however, is an altogether different story. Typical best numbers amount to peak currents of $1 \mathrm{kA}$ at $100 \mathrm{fs}$ bunch lengths, i.e., $\sigma_{z} \approx$ $30 \mu \mathrm{m}$, combined with an rms energy spread of the order of $100 \mathrm{keV}$, i.e., $\sigma_{p_{z}} / m c \approx \sigma_{E} / m c^{2} \approx 0.2$, implying an increase of the longitudinal emittance, and therefore a decrease of the $6 \mathrm{D}$ brightness, by 6 orders of magnitude. Clearly, the challenge that lies ahead is battling the increase in longitudinal emittance during acceleration.

\section{BRIGHTNESS DEGRADATION MECHANISMS}

In general, the $6 \mathrm{D}$ rms brightness is reduced during acceleration due to deformations of the phase-space distribution of the bunch, caused both by the external fields and the space-charge self-fields. There are two main classes of such deformations:

The first is reasonably well described by the term curvature, and is caused by all kinds of nonlinear effects such as nonlinear space-charge forces and, for example, spherical aberrations of electromagnetic lenses. Although the local phase-space density is conserved, curvature causes the averaged quantities, i.e., the rms emittances, to increase. Under some conditions two nonlinear effects can be made to cancel each other [18], but in general it causes unrecoverable degradation of the rms $6 \mathrm{D}$ brightness $B$.
The second class of deformations are correlations in more than two dimensions, such as a slope of the phasespace density distribution in the $x$ - $p_{x}$ projection that varies as a function of $z$. The latter is a well known and an undesired side effect of space-charge forces inside a uniformly filled cylinder and it results in a larger overall (projected) emittance and hence a decreases in rms brightness. To what extent such correlations are a limiting factor is very much application dependent. An application that mainly depends on slice emittance, such as a SASE-FEL, will not be affected. Furthermore, emittance compensation schemes $[8,17]$ can be very effective in reducing this effect. However, in general these correlations are undesired.

There is only one type of 3D charge density distribution that does not suffer from space-charge related deformations in phase space. These are uniformly filled ellipsoids, also known as waterbag bunches [2]. Such objects have linear space-charge fields in all three coordinates. Under the influence of their internal space-charge forces they will deform as a function of time, but their shape will always remain a uniformly filled ellipsoid as long as the external forces are linear. In theory, waterbag bunches offer the possibility to maintain the $6 \mathrm{D}$ brightness of bunches produced by rf photoguns at their initial value; potentially a gain of orders of magnitude in brightness is possible compared to state-of-the-art rf photoguns. However, as we will see in the remainder of this paper, waterbag bunches in practice have slightly soft edges and-more importantly-external forces are never truly linear. Chromatic aberrations of the focusing system, path-length differences, the effects of fringe fields and nonlinear space-charge effects resulting from these nonideal conditions all need to be taken into consideration before it is known to what extent waterbag bunches can be manipulated, e.g., compressed, in practice.

\section{WATERBAG BUNCHES}

A practical way to realize a uniformly filled, 3D ellipsoidal bunch is by starting out with a charge density distribution on the cathode surface given by [4]

$$
\rho(r, z)=\sigma_{0} \sqrt{1-(r / R)^{2}} \alpha(z),
$$

where $\sigma_{0}=3 \mathrm{Ne} /\left(2 \pi R^{2}\right)$ is the surface charge density at the center, $N$ the number of electrons, and $\alpha(z)$ is a longitudinal distribution function, which ideally approaches a delta function. A sufficiently flat initial distribution can be realized by photoemission from copper with a transversely shaped femtosecond UV laser pulse, assuming response times of the order of $10 \mathrm{fs}$. If an acceleration field $E_{0}$ is applied such that

$$
\frac{e E_{0} \tau_{l}}{m c} \ll \frac{\sigma_{0}}{\varepsilon_{0} E_{0}} \ll 1,
$$

where $\tau_{l}$ is the duration of the photoemission process, i.e., 
approximately the laser pulse length, then the pancake bunch automatically evolves, driven by its internal spacecharge forces, into a full-fledged 3D waterbag distribution.

In Ref. [4] we showed by GPT simulations that a $100 \mathrm{pC}$ bunch created by photoemission with a properly shaped $1 \mathrm{~mm}$ radius, $30 \mathrm{fs}$ laser pulse will indeed evolve into a 3D waterbag distribution, with perfectly linear momentumposition phase-space correlations. In these first simulations a constant and uniform $100 \mathrm{MV} / \mathrm{m}$ acceleration field was assumed. As a natural extension we showed in [6] that waterbag bunches can be closely approximated in a "standard" 1.5 cell rf photogun, taking into account realistic accelerating fields, space-charge forces, image charges, and magnetic focusing. The combined effect of image charges and these other nonideal, realistic fields resulted in a slightly egg-shaped bunch. However, despite this small deformation, the initial transverse emittance was fully recovered. In addition, the bunch showed an almost perfect linear longitudinal momentum-position phase-space correlation, with a width in the order of only $10 \mathrm{fs}$. This suggests that a linear compression scheme can compress these bunches to multi-kA peak currents with rms normalized transverse emittances below $1 \mu \mathrm{m}$.

\section{WATERBAG COMPRESSION}

The current paradigm in accelerator physics is that to produce short, $100 \mathrm{fs}$, high-brightness kA electron bunches, it is essential to start with multi-ps bunches and compress them after acceleration to high energies where detrimental space-charge effects are sufficiently suppressed [19]. In this section we show that waterbag bunches, because of their linear internal fields, allow longitudinal phase-space manipulation at subrelativistic or mildly relativistic energies. A straightforward approach is presented that breaks with the current paradigm and achieves efficient compression of high-brightness waterbag bunches in a simple but realistic setup at only $1 \mathrm{MeV}$.

To ballistically compress a bunch with length $L$ over a distance $d$, the required velocity difference $\Delta v$ between the head and tail particle is given by $\Delta v=v L / d$. From the definition of the Lorentz factor and the kinetic energy of a particle given by $E=(\gamma-1) m c^{2}$ it immediately follows that

$$
\Delta v=\frac{1}{v \gamma^{3}} \frac{\Delta E}{m}
$$

where $\Delta E$ is the kinetic energy difference between the head and tail of the bunch. The $\gamma^{3}$ factor in the denominator of Eq. (8), in literature sometimes referred to as the longitudinal mass factor, makes ballistic compression particularly efficient at low energies because far less energy spread is required for the same amount of compression.

\section{A. Setup}

Shown in Fig. 1 is the system under investigation. It consists of a $\frac{1}{2}$-cell S-band rf cavity, illuminated by a femtosecond UV photoexcitation laser with a transverse intensity profile as given in Eq. (6), a 2-cell S-band booster compressor, and four solenoids. To guarantee fully cylindrically symmetric fields, axial incoupling, pioneered by DESY, and with dimensions presented in $[20,21]$ is used.

The amplitude of the electric accelerating field determines the total amount of charge that can be extracted; see Eq. (7). However, a high energy is not desirable for ballistic bunching because of the $\gamma^{-3}$ dependency in Eq. (8). To get the best of both worlds, we decided to start with a $100 \mathrm{MV} / \mathrm{m} \mathrm{S}$-band cavity, only a half-cell in length to limit the final energy to $1 \mathrm{MeV}$, i.e., $\gamma \approx 3$. Because the waterbag shape prevents rms brightness degradation, we can use a simple drift space to let the bunch expand to a few ps, which is sufficiently long for S-band compression.

A 2-cell $100 \mathrm{MV} / \mathrm{m}$ cavity, fully analogous to the last two cells of the $2 \frac{1}{2}$-cell rf-photogun described in [12], is used as combined booster and compressor. It increases the energy to about $3.5 \mathrm{MeV}$, while simultaneously reversing
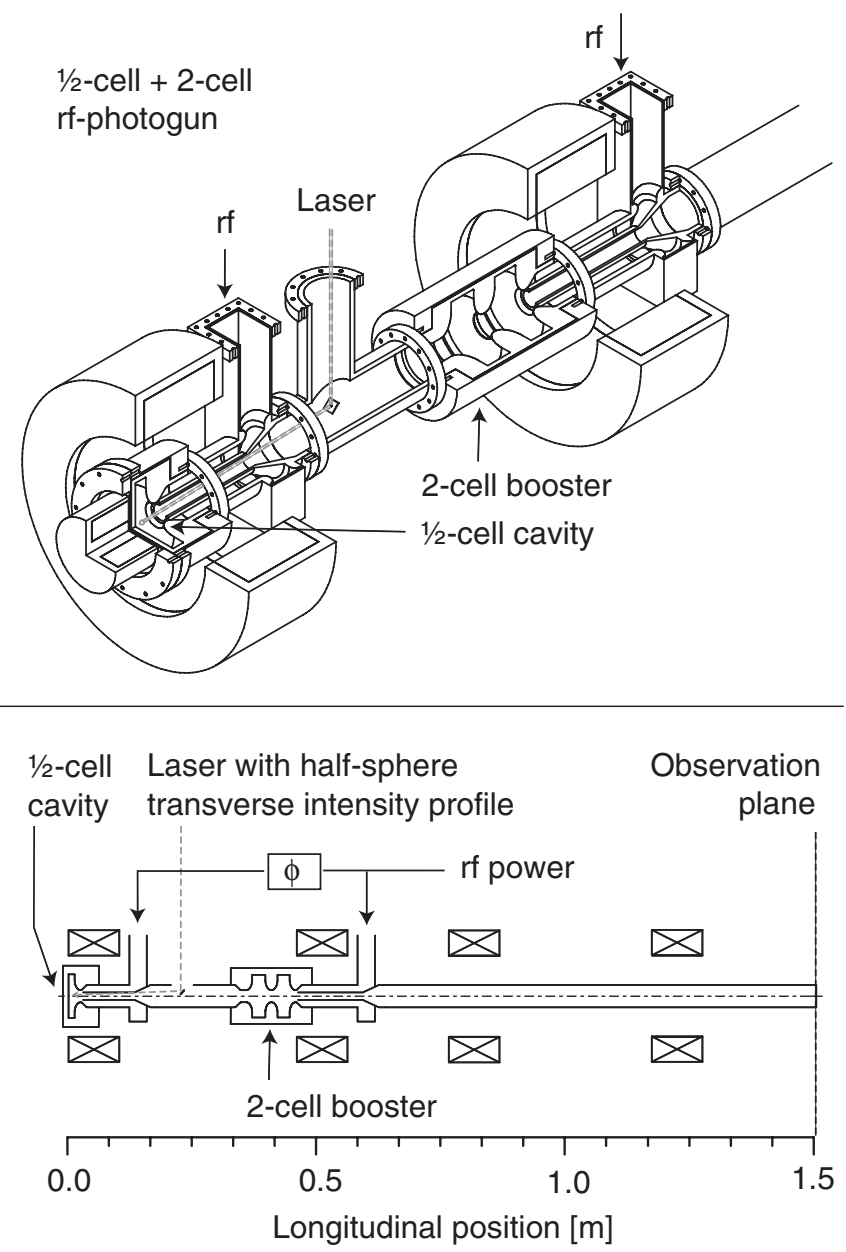

FIG. 1. Schematic of the proposed setup. 
the slope in longitudinal phase space from space-charge expansion to ballistic compression. The total power consumption of the combined rf cavities is $10 \mathrm{~W}$.

Compression is an intrinsically nonlinear process because the relation between velocity and energy is not linear. In addition, both off-crest acceleration and the energy dependence of the cavity transit times result in a nonlinear component in transverse phase space. The phase of the rf booster has been set such that all nonlinear effects cancel each other to second order, resulting in minimum bunch length at the point of maximum compression, at $z=1.5 \mathrm{~m}$. The transverse dynamics is governed by four solenoids and a bucking coil, all with fields below $0.2 \mathrm{~T}$.

\section{B. Numerical modeling}

The GPT code [5] was used for the detailed particle tracking simulations of the setup under investigation. The relativistic equations of motion of typically $100 k$ to $1 \mathrm{M}$ sample particles are integrated through the combined fields of the rf cavities, the solenoids and space charge. This approach includes path-length differences, nonlinear external fields, and nonlinear space-charge forces. The integration steps of the embedded 5th order Runge-Kutta integrator [22] are automatically chosen, based on a maximum tolerance of $10^{-5}$ for the dimensionless momentum coordinates. This results in step sizes of a few fs near the cathode region, rapidly expanding to the ps regime in the cavities and as large as a ns in the drift spaces. The multidimensional solver, combined with hierarchical data analysis, has been used to automate the task of finding the final solenoid and rf phase settings presented in this paper. The standing-wave field pattern in both cavities is calculated with the SUPERFISH set of codes [23], and fed into GPT as cylindrically symmetric field maps with $0.25 \mathrm{~mm}$ spacing in both the $z$ and $r$ directions. The entry and exit fields are included and truncated at $10^{-6}$ times the maximum field. The magnetostatic solenoid fields are based on an analytical expression for the field on-axis, and 5th order expansion for the off-axis fields. Although GPT allows all beam line components to be positioned arbitrarily in 3D space to study tolerances and the effect of misalignment, the presented simulations have all beam line components exactly on axis. All space-charge forces are calculated in 3D with a PIC approach, where Poisson's equations are solved in the rest frame of the bunch. The effect of image charges is included with a Dirichlet boundary condition at the $z=0$ plane. Technical details related to the adaptive meshing strategy and the multigrid solver, tailor made for bunches with extreme aspect ratios, can be found in [24].

\section{Longitudinal compression}

The overall multiparticle GPT simulation results of a $100 \mathrm{pC}$ bunch with $1 \mathrm{~mm}$ initial radius and a transverse profile given by Eq. (6) are shown in Fig. 2. The particles are injected on crest of the rf field at the cathode surface to

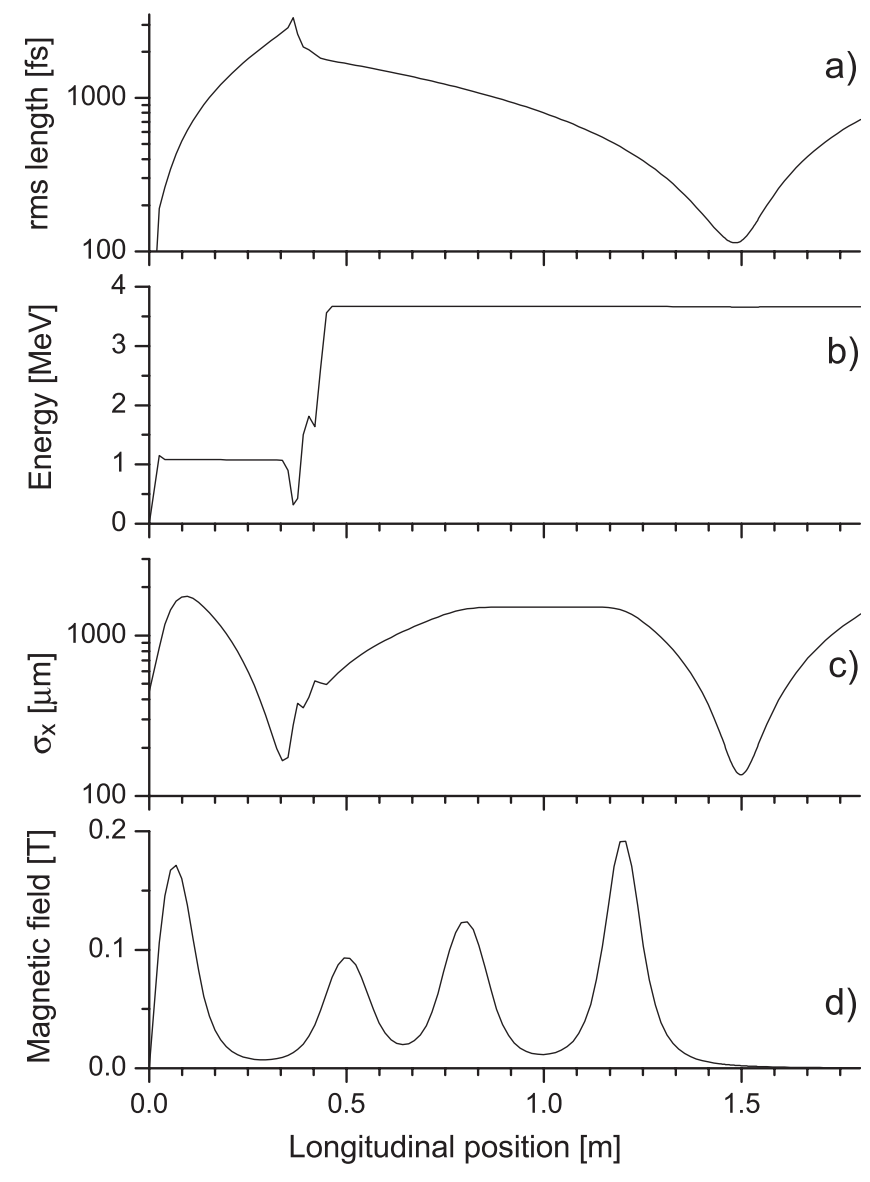

FIG. 2. GPT simulation results of (a) rms bunch length, (b) energy, and (c) rms transverse bunch size $\sigma_{x}$ as a function of position $z$; (d) on-axis magnetic field-profile.

minimize the effect of image charges, with a Gaussian temporal profile of 30 fs full width at half maximum (FWHM). As shown in (a), this bunch expands rapidly due to space-charge forces to over $2 \mathrm{ps}$, to be compressed down to $100 \mathrm{fs}$ rms at the longitudinal focus at $z=1.5 \mathrm{~m}$. The final energy, see (b), is $3.7 \mathrm{MeV}$. The sharp decrease in energy at the entrance of the booster is used to correct for second-order effects in longitudinal phase space. The beam envelope (c) is mainly determined by the magnetic profile (d). An intermediate waist near the two-cell booster compressor minimizes the effect of nonlinear coupling between transverse coordinate due to the acceleration field at the entrance and exit. Downstream, a transverse focus $\sigma_{x}=$ $\sigma_{y}=140 \mu \mathrm{m}$ is created at exactly the same position as the longitudinal focus.

Detailed results at the combined longitudinal and transverse focus at $z=1.5 \mathrm{~m}$ are shown in Fig. 3. The proposed setup compresses an ellipsoidal bunch to a peak current of more than $550 \mathrm{~A}$ and a FWHM pulse duration of $130 \mathrm{fs}$ with a setup comparable in size and complexity to a single rf photogun. The longitudinal phase space at the focal plane is fully determined by an accumulation of nonlinear 

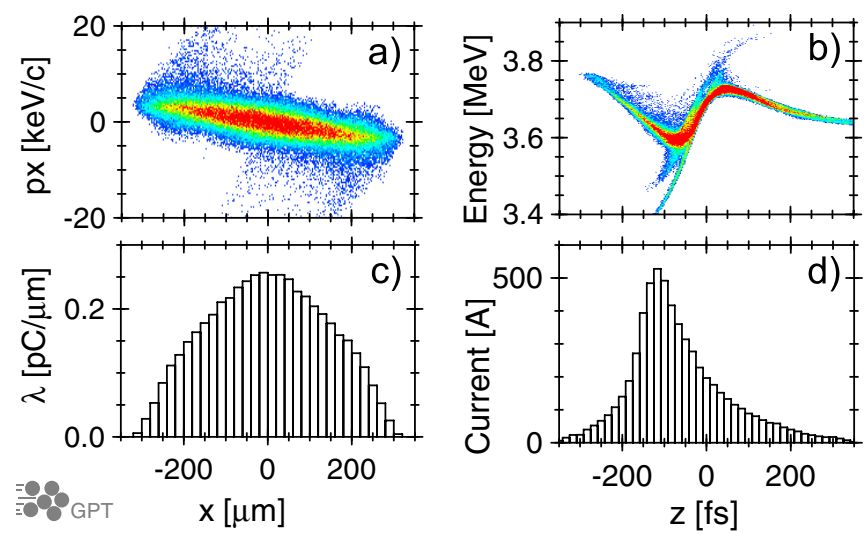

FIG. 3. (Color) (a) Transverse phase space, (b) longitudinal phase space, (c) transverse line-charge density, and (d) current profile at the combined longitudinal and transverse focal plane at $z=1.5 \mathrm{~m}$, obtained with $100 \mathrm{k}$ multiparticle GPT simulations. The online colors indicate particle density and range from blue (low density), via green and yellow to red (highest density).

effects, and no trace remains of the initially perfect position-energy correlation. In the transverse direction, however, the ellipsoidal shape is very well maintained, as can be seen by the line-charge density profile $\lambda(x)=$ $\int \rho(\vec{r}) d y d z$, which has the desired parabolic shape. The rms emittance of $0.7 \mu \mathrm{m}$ is very close to the initial thermal emittance of $0.5 \mu \mathrm{m}$. This amounts to a transverse brightness of $26 \mathrm{~A} \mathrm{~m} \mathrm{~m}^{-2} \mathrm{sr}^{-1}$, exceeding the $4 \mathrm{MeV}$ ballistic compression scenario presented in [9] by orders of magnitude.

We would like to stress that simultaneous longitudinal and transverse compression is just one of many interesting scenarios. Obviously there are many tradeoffs possible in the optimization of either the transverse or the longitudinal phase space. If, for example, the transverse size is not a constraint, switching off the last solenoid eliminates pathlength differences between the outer and inner particles due to transverse focusing. In this way $2 \mathrm{kA}$ peak currents with a FWHM pulse duration of only $30 \mathrm{fs}$ can be attained with $\sigma_{x}=\sigma_{y}=2 \mathrm{~mm}$.

\section{Energy spread reduction}

Ballistic bunching is based on velocity differences between the head and tail of the bunch and this in turn translates into undesired energy spread. A clear advantage of compression of ellipsoidal bunches at mildly relativistic energies is that it significantly reduces the required absolute energy spread compared to compression at high energy; , see Eq. (8). To further decrease the energy spread, we make use of the fact that ballistic bunching of ellipsoidal bunches requires a linear negative correlation in position-momentum space, as opposed to space-charge forces which introduce a linear positive correlation.

The rate of change of the slope $d p_{z} / d z$ of a waterbag distribution in longitudinal phase space due to space- charge forces is given by

$$
\frac{d}{d t} \frac{d p_{z}}{d z}=\frac{e \rho_{0}}{\epsilon_{0}} M_{z}
$$

with $\rho_{0}$ the central charge density and $\epsilon_{0}$ the permittivity of vacuum. For cylindrically symmetric bunches $M_{z}=[(1+$ $\left.\Gamma) / \Gamma^{3}\right](\Gamma-\arctan \Gamma) \quad$ with the eccentricity $\Gamma=$ $\sqrt{\sigma_{x}^{2} / \sigma_{z}^{2}-1}$, see [1]. Because $M_{z}$ depends on the transverse size $\sigma_{x, y}$ it is in theory possible to set the beam envelope such that all correlated energy spread required for the bunching process is canceled by the integrated effect of the space-charge forces. This implies simultaneous reduction of $\sigma_{z}$ and $\sigma_{p_{z}}$. Liouvilles theorem dictates that this is possible only if one starts with a sufficiently narrow distribution in position-momentum space, and hence a very low longitudinal emittance, a key feature of waterbag bunches.

To demonstrate the reduction of $\sigma_{p_{z}}$ due to space-charge forces in our example setup, a transverse focus of $\sigma_{x, y}=$ $140 \mu \mathrm{m}$ was chosen. This value results in a sharp decrease in energy spread as shown in Fig. 4(a). Interestingly, the average kinetic energy of the bunch decreases as well, as shown in Fig. 4(b). This may sound counterintuitive, as a single particle does not change kinetic energy in a magnetic field. However, the dynamics of a whole bunch of charged particles compressed inside a magnetic field is governed by conservation of energy. Compression results in an increase in potential energy that is matched by an equal decrease in average kinetic energy. On $\mathrm{MeV}$ scales this is a negligible effect. It nevertheless reduces the absolute energy spread by a factor of 3 to $\sigma_{E}=40 \mathrm{keV}$, undoing the energy spread introduced to compress the bunch. This translates into a $6 \mathrm{D}$ brightness of $B=$

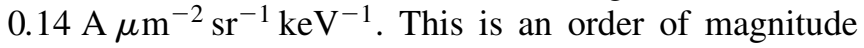
better than the best velocity bunching results presented in
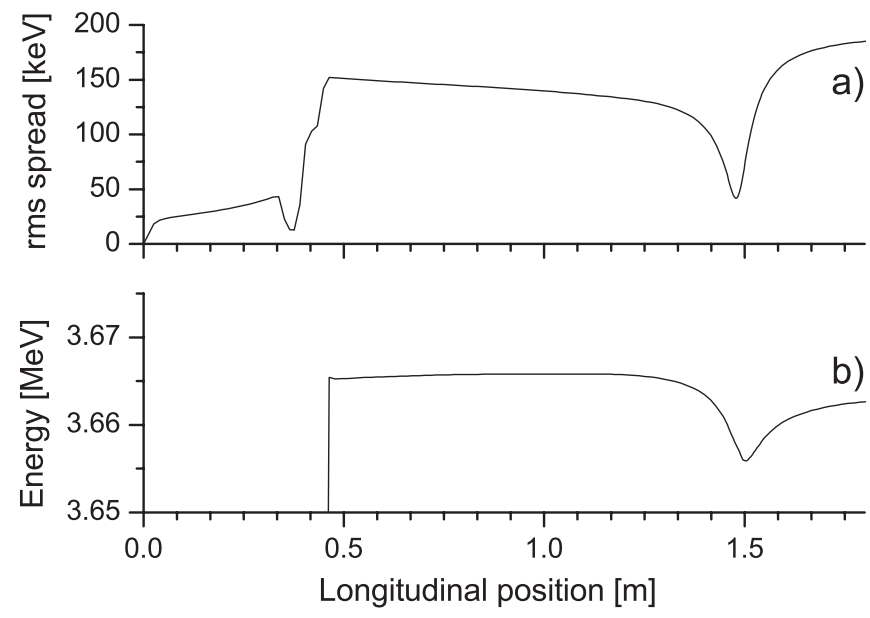

FIG. 4. GPT simulation result for (a) energy spread and (b) average kinetic energy as a function of longitudinal position. 
Ref. [9] due to a significantly smaller longitudinal phase space.

\section{CONCLUSION}

Ideally, the brightness of ellipsoidal bunches does not deteriorate in a linear transport and focusing system. This makes them ideal objects for both longitudinal and transverse compression. In practice, however, a large number of effects distort the ideal ellipsoidal shape: At creation, the ellipsoid is not ideal because of image charges at the cathode and the finite laser duration convoluted with the response time of the cathode. Downstream, the inevitable energy spread results in chromatic aberrations. Furthermore, the entry and exit fields of the rf cavities are intrinsically nonlinear. Finally, any transverse excursions, in all rf cavities and at the transverse focal point result in path-length differences between the outer and inner particles that play a significant role for femtosecond bunches. All these effects are aggravated by the fact that once the ellipsoidal shape is distorted, nonlinear space-charge forces come into play again which further deteriorates the rms brightness.

In theory, waterbag bunches allow very efficient phasespace manipulation at low energy, in the $1 \mathrm{MeV}$ range, without the detrimental effects of nonlinear space-charge forces normally associated with the $1 \mathrm{MeV}$ regime. To test to what extent ellipsoidal bunches can be created and compressed in a realistic setting, we investigated a compact setup consisting of a $\frac{1}{2}$-cell rf cavity, a 30 fs UV laser photoexcitation laser with proper transverse shaping, and a 2-cell booster compressor. Detailed GPT simulations predict $2 \mathrm{kA}, 30 \mathrm{fs}$ bunches, based on realistic fields and including the effect of nonlinear space-charge forces and image charges. Interestingly, an even higher rms brightness can be obtained if the bunches are simultaneously focused to a transverse size of $\sigma_{x}=140 \mu \mathrm{m}$. In that case, the correlated energy spread required for ballistic bunching is fully canceled by longitudinal space-charge forces. The final emittance is reduced to $0.7 \mu \mathrm{m}$ combined with a remaining rms energy spread of only $40 \mathrm{keV}$, at the expense of peak current which drops to $500 \mathrm{~A}$.

The high peak current, short pulse duration and focusability make compressed ellipsoidal bunches ideal for the production of broadband, high-power terahertz radiation [11] and pulsed radiolysis [14]. Furthermore, they have ideal parameters to inject into a controlled plasmawakefield accelerator [12]. Many applications, however, like Compton backscattering to generate coherent hard $\mathrm{x}$-rays and the x-ray free electron laser [19], require much higher energies. Since there is no objection to shifting the longitudinal focus to the downstream end of an additional linac, low-energy ballistic compression of waterbag bunches might be an interesting alternative to high-energy magnetic compression techniques.
Space-charge induced energy spread reduction is essentially the time-reversed process of the creation of an ellipsoid at the cathode. This in turn implies that in theory waterbag bunches make it possible to image the cathode at the longitudinal focus, while maintaining the full 6D rms brightness. This would result in nm long, sub-fs, bunches with an energy spread below $1 \mathrm{eV}$. Clearly, a tremendous reward is waiting if we are able to transport and compress ellipsoidal bunches in more linear external fields.

[1] O. D. Kellogg, Foundations of Potential Theory (SpringerVerlag, Berlin, 1929).

[2] I. M. Kapchinskii and V. V. Vladimirskii, in Proceedings of the International Conference on High Energy Accelerators, CERN, Geneva (Scientific Information Service CERN, Geneva, 1959), p. 274.

[3] L. Serafini, in Towards X-Ray Free Electron Lasers: Workshop on Single Pass, High Gain FELs Starting from Noise, Aiming at Coherent X-Rays, edited by Rodolfo Bonifacio and William A. Barletta, AIP Conf. Proc. No. 413 (AIP, New York, 1997), p. 321; in Advanced Accelerator Concepts, edited by J.S. Wurtele, AIP Conf. Proc. No. 279 (AIP, New York, 1993), p, 645.

[4] O.J. Luiten, S. B. van der Geer, M.J. de Loos, F. B. Kiewiet, and M.J. van der Wiel, Phys. Rev. Lett. 93, 094802 (2004).

[5] http://www.pulsar.nl/gpt

[6] O. J. Luiten, S. B. van der Geer, M.J. de Loos, F. B. Kiewiet, and M.J. van der Wiel, in Proceedings of the European Particle Accelerator Conference, Lucerne, 2004 (EPS-AG, Lucerne, 2004), p. 725.

[7] C. Limborg-Deprey and P. R. Bolton, Nucl. Instrum. Methods Phys. Res., Sect. A 557, 106 (2006).

[8] J. B. Rosenzweig, A. M. Cook, R. J. England, M. Dunning, S. G. Anderson, and M. Ferrario, Nucl. Instrum. Methods Phys. Res., Sect. A 557, 87 (2006).

[9] S. G. Anderson et al., Phys. Rev. ST Accel. Beams 8, 014401 (2005).

[10] K. T. McDonald, IEEE Trans. Electron Devices 35, 2052 (1988).

[11] G. P. Gallerano and S. Biedron, in Proceedings of the 2004 Free Electron Laser Conference, Trieste, Italy, 2004 (Sincrotrone Trieste, Trieste, 2004), p. 318, available online at http://www.JACoW.org.

[12] W. H. Urbanus, W. van Dijk, S. B. van der Geer, G. J. H. Brussaard, and M. J. van der Wiel, J. Appl. Phys. (to be published).

[13] T. C. Chiou and T. Katsouleas, Phys. Rev. Lett. 81, 3411 (1998).

[14] Y. Muroya, T. Watanabe, G. Wu, X. Li, T. Kobayashi, J. Sugahara, T. Ueda, K. Yoshii, M. Uesaka, and Y. Katsumura, Radiat. Phys. Chem. 60, 307 (2001).

[15] B. J. Siwick, J. R. Dwyer, R. E. Jordan, and R. J. D. Miller, Science 302, 1382 (2003).

[16] C. A. Brau, The Physics and Applications of High Brightness Electron Beams, edited by J. Rosenzweig, G. Travish, and L. Serafini (World Scientific, Singapore, 2003), p. 20. 
[17] B. Carlsen, Nucl. Instrum. Methods Phys. Res., Sect. A 285, 313 (1989).

[18] S. B. van der Geer, M. J. de Loos, J. I. M. Botman, O. J. Luiten, and M. J. van der Wiel, Phys. Rev. E 65, 046501 (2002).

[19] DESY-TTF, Hamburg, Germany, http://tesla.desy.de.

[20] S. B. van der Geer and M.J. de Loos, Ph.D. thesis, Eindhoven University of Technology, 2001.

[21] F. B. Kiewiet, Ph.D. thesis, Eindhoven University of Technology, 2003.
[22] W. H. Press et al., Numerical Recipes, The Art of Scientific Computing (Cambridge University Press, Cambridge, England, 1992), 2nd ed.

[23] J.H. Billen and L.M. Young, POISSON SUPERFISH, Los Alamos National Laboratory Report No. LA-UR-961834.

[24] G. Pöplau, U. van Rienen, S. B. van der Geer, and M. J. de Loos, IEEE Trans. Magn. 40, 714 (2004). 